\title{
GLAID: Designing a Game Learning Analytics Model to Analyze the Learning Process in Users with Intellectual Disabilities
}

\author{
Ana R. Cano ${ }^{1(\bowtie)}$, Baltasar Fernández-Manjón ${ }^{1}$, \\ and Álvaro J. García-Tejedor ${ }^{2}$ \\ ${ }^{1}$ Universidad Complutense de Madrid, Madrid, Spain \\ anarcano@ucm.es, balta@fdi.ucm.es \\ ${ }^{2}$ Universidad Francisco de Vitoria, Madrid, Spain \\ a.gtejedor@ceiec.es
}

\begin{abstract}
Educational Games are increasingly popular in teaching as they have proven to be effective learning tools. Educational videogames are beneficial for all kind of students but we think they are especially suited for users with intellectual disabilities due to the opportunity of tailoring the content to their in-game performance. Adapting the game experience to the cognitive and learning abilities to this type of students also make videogames a powerful source of learning data. In this paper we introduce the GLAID (Game Learning Analytics for Intellectual Disabilities) Model, a theoretical adaptation of a more general analytics framework. It describes how to collect, process and analyze videogame interaction data in order to provide an overview of the user learning experience, from an individualized assessment to a collective perspective. But to obtain these goals it is necessary to take into account the restrictions and special needs of users with intellectual disabilities both in the learning design and in translating them into game mechanics and the corresponding observables that will be collected for the subsequent data analysis. We conclude with a discussion and considerations about the model and future steps to follow in our investigation.
\end{abstract}

Keywords: Serious games · Intellectual Disabilities · Game Learning Analytics · Analytics maturity framework · Educational games · Down syndrome $\cdot$ Autism spectrum disorders

\section{Introduction}

The impact of new technologies on education is changing the way teaching is occurring in the classroom as technology can ease a more active approach of the students in their own learning process. Videogames are increasingly used in education because of their capacity to engage the user, making the learning experience a more dynamic activity where the student needs to make decisions and learn from their effects. Educational videogames are attractive and engaging to students. Usually, videogames are designed to adapt the game experience to different users, offering different levels of difficulty or tailoring the content to the in-game performance [1]. 
But videogames can also be used as research and/or measurement tool [2], giving educators the opportunity of collecting information about the user's behavior and interactions within the game. A game, due to its highly interactive nature, can generate larger amounts of data than other tools [3]. When using an educational videogame, it is possible to record all the interactions of the player during the game session in order to track the evolution of the student and use that data to better understand or to improve the learning process. The processing and analysis of all that interaction data and transform it into valuable information for the student's assessment is the target that we pursue with the application of our model. The emerging field that address these aspects is called Learning Analytics (LA), defined as "the measurement, collection, analysis and report data about learners and their context, for purposes of understanding and optimizing learning and the environments in which it occurs" [4].

The application of LA in educational videogames is called Game Learning Analytics (GLA) and combines the educational goals of LA with the tools and technologies from Game Analytics [5]. GLA is specifically conceived to facilitate the process of analyzing significant variables or observables (like playing time, level changes or goals achieved) to better understand how users learn and to provide a better assessment.

All these GLA processes of automatic collection and analysis of interaction data become even more important when the students are people with special needs. For instance, intellectual disabilities cause several communication problems [6] that can prevent educators to understand the way students are processing the information and the effectiveness of their learning experience. This makes very difficult to use standard game evaluations that are usually based on pre-post forms that users fill before and after playing the game.

In this article we introduce the GLAID Model as a variation of the classical Analytics Maturity Framework defined by the Big Data industry [7]. This model is intended to describe the different stages of data that educators and game designers can analyze from students with intellectual disabilities when they are playing a learning video game. The last section of the article discusses recommendations and next steps to follow this line of research.

\section{The Importance of Game Design While Working with Users with Intellectual Disabilities: Restrictions, Requirements and Game and Learning Design}

Users with intellectual disabilities have problems not only interacting with the game but also communicating the difficulties found while they are playing. As a result, we cannot fully rely on their testimony when we are evaluating their learning experience within the game.

Some of these communication problems can be troubles in ordering thoughts and language in a 'logical' layout; problems learning to listen and taking turns in conversations; difficulties using communication in an interactive sense or problems relating objects and actions to spoken or written words [8]. LA can help to address this issue by 
evaluating the adequacy of the learning experience to this kind of users through an indirect way: a data-driven analysis instead of a subjective responses analysis.

Creating an adequate learning videogame suitable for users with intellectual disabilities requires to have in mind all the player special needs that can affect the game mechanics. Defining the user characteristics and cognitive restrictions and translating them into formal user requirements is a pre-requisite to build an inclusive game design. That information would be used in the learning design and in the identification of information about the learning process that will be collected and analyzed. Therefore, a good design is essential to obtain meaningful data for a reliable assessment.

User features, either cognitive, psychological or motor, determine the learning methods to design the mechanics of the game (like the possibility of select the difficulty level adapted to each user or the use of a dynamic pop-up aid) and the interpretation of the collected interaction information.

Some of the user's restrictions and characteristics that designers should consider during the development of a learning game, specifically for players with intellectual disabilities, divided by psychological areas can be [9-11]: (Table 1)

Once we know what is distinctive in this type of users, the second step is to translate these characteristics into formal user requirements. Having specific cognitive accessibility guidelines, like the ones that appear in the standard WCAG 2.0 [12] or in the GAG [13], can be used to streamline the design process.

Table 1. User characteristics related to intelligence, memory and perception, personality and motor skills

Intelligence, Memory and Perception

- Visual perception and visual retention better than hearing

- Procedural memory good enough for playing

- Short Memory limitation. Limitation in the number of instructions/numbers that they can handle at the same time (3 sequential instructions and 3-6 digits)

- Difficulty sustaining attention during long periods of time

- Mild intellectual deficiency

- Problems of understanding the information

- Difficulty in the process of abstraction, conceptualization, generalization and learning transfer

Personality

- Limited initiative

- Persistence of behavior and resistance to change

- Few response capacity and reaction to the environment

- Poor social and collaboration skills (depending on the disability)

Biological and Motor Skills

- Listening and sight problems

- Heart diseases

- Clumsiness in motor skills (gross and fine)

- Poor coordination 
The application of the standards and a deep knowledge of the idiosyncrasy of the user and can minimize the number of backward changes in an agile videogame development.

\section{The Game Learning Analytics for Intellectual Disabilities Model}

Once known the user unique characteristics and how this affects the translation of the learning design into game mechanics, next step would be working on creating an effective game design suitable for the users. The development should also include the traces of information needed for the students' assessment inside the code.

Since there are not reference guides to include GLA in videogames for players with intellectual disabilities, we adapted a classical analytics framework used in the business industry [7] to create our own model. We called it Game Learning Analytics for Intellectual Disabilities (GLAID) Model. This model describes what type of data would be useful to collect, group and analyze to provide an overview of the learning experience of each user from an individualized assessment to a collective perspective. The last step of the model is the prediction of the potential behavior of new players within the game and the evolution of the learning progress of existing users, attending to the learning experience, based on other users' historical data.

In this section we describe each step of the model and its correlation with each phase of the analytics framework. (Figure 1).

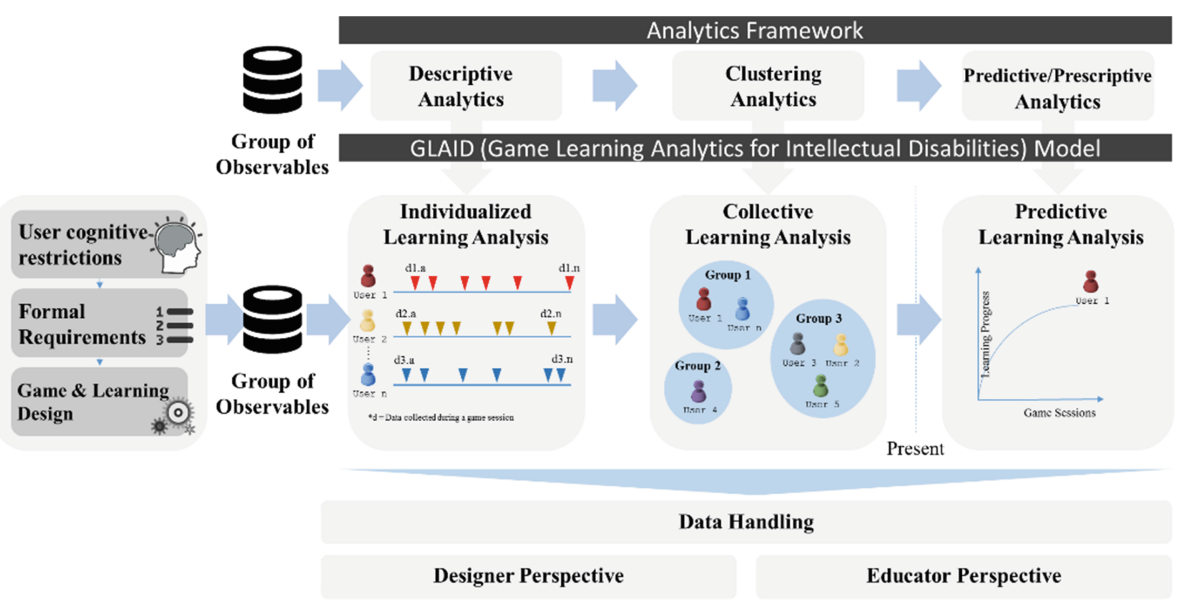

Fig. 1. Analytics maturity stages of the GLAID 


\subsection{Individualized Learning Analysis}

The first step of the GLAID Model is the Individualized Learning Analysis. This phase corresponds with the Descriptive Analytics stage. The goal in this phase is to describe and analyze historical learning data from the student's perspective.

In this phase, educators and game designers collect all the interactions of the user within the game to analyze which data are/are not relevant for future assessment. The real challenge of this stage is taking into account all the users' special requirements, the learning design and the game design to select the signals or game observables (i.e. variables) that can give useful information about the user's learning behavior. Once the variables are identified, educators can describe the trends of each student for a personalized assessment. The better the design is adapted to the cognitive features of the user, the easier the assessment can be settled.

Typical data to collect and analyze in this stage can be:

- Timestamps. Generating a trace with data about when the user starts, ends, quits the game or stays inactive [14] provide relevant information about the level of engagement of the player, the effectiveness of the game design and the evolution of the learning process.

- Level changes. Videogames are structured by levels, screens, missions or chapters. Tracing each level status during a certain period of time can help educators and researchers to figure out if the student is understanding the purpose of the game and, as a result, if the learning experience is being successful.

- Achievements vs Fails. Tracing the evolution of the ratio achievements/fails during several consecutive game sessions is a good indicator about how the user's learning experience is progressing.

- User interactions. Any user interaction like number of clicks, heatmaps, number of times that the help button is pressed, time between clicks, number of attempts completing a task, etc. can be potentially useful for the assessment, giving a more comprehensive overview about the behavior of the student inside the game.

Data handling, visualization and interpretation is described below, in its own separate section.

\subsection{Collective Learning Analysis}

The next level of maturity in GLAID is the Collective Learning Analysis where the researcher and educator could identify causes of trends and learning outcomes of a group of users. In this stage, a collection of reports and statistics are generated from the perspective of a class, a group, or even more granular, a certain type of disability. As an example, we can obtain relevant information about the level of understanding of users with Autism Spectrum Disorder (ASD) tracing data and patterns throughout their common conducts inside the game.

The purpose of the clustering analysis is to diagnose learning outlines in different segments to obtain conclusions about how the learning process of different users with a common pattern has occurred. The game designer can obtain information about the 
adequacy of the game's mechanics to certain types of disabilities or groups of users (i.e. players with Down Syndrome between 10 to 15 years old that play twice a week obtain better results that those with ASD in the same range of age and playing three times a week). It can also be used to test the efficacy of some of the decisions made on the learning design.

Clustering methods or any other machine learning techniques can be applied in this stage to uncover patterns in the data that provide additional unknown information from the previous stage of the model.

\subsection{Predictive/Prescriptive Learning Analysis}

The Predictive Learning Analysis is the third and last phase of the model. It embodies a group of statistical techniques and analyzes current and historical data (obtained in the descriptive analysis and clustering analysis/machine learning phase) to make predictions about future learning outcomes [15]. To make accurate predictions it is necessary to collect data from a large population of users (which could be problematic in this case due to the specificities of the target users).

\subsection{Data Handling}

The information described above can be automatically collected and displayed in reports, but data visualization would differ depending on the recipient of the report and its final purpose.

Ideally, educators and researchers should be able to get insight about how the user has been playing within the game at a glance of the report, without directly observe the user playing. The analysis of the data collected can be done through two different perspectives:

1. Game Designer's perspective. The game designer is interested in collecting and analyzing all the states that the user can reach at any moment within a game session. The purpose of this analysis is to determine if the gameplay and mechanics designed are properly adapted to the user and effective for their cognitive, psychological and motor capabilities.

2. Educator's perspective. The educator is focused on each user learning experience and this report should provide the relevant data about it. Videogames can be considered as a new learning tool that offer useful information that may not be collected using traditional methodologies, like the exact number of failures/achievement in each learning session. Ideally this report should inform the educator if the user is learning or struggling with the game or at least help them to identify users that may need some extra support. 


\section{Conclusions and Future Work}

Our paper proposes a model called GLAID (Game Learning Analytics for Intellectual Disabilities) that aims to incorporate different levels of data treatment into a learning game development for users with intellectual disabilities, like Down syndrome, mild cognitive impairments or Autism Spectrum Disorders.

Although the model proposed is theoretical and should apply to any development, some considerations about it deserve discussion: The game engine used for the development should allow collecting the observables to analyze the learning process. We are currently working on the development of a GLA module for Unity3D engine as part of the H2020 RAGE project that can address this issue and can work as an example to replicate for other game engines. Once this module is working, the application of the GLAID model to the data handling will be easier. But this still requires that the learning design and game mechanics should be specifically designed for the gamers that are going to use the videogame as a learning tool. As long as there is a wide range of cognitive conditions, it is not possible to fully standardize the game design process but the application of this framework step by step should provide feedback depending on the commonalities and divergences found in the target audience. The help of experts (not only psychologists, social workers or specialized teachers but also game and interactions designers) is essential to obtain a truly valuable learning game. The application of our model is focused on users with intellectual impairments, which struggle with several communication problems making the process of obtaining feedback of the player more complex and less reliable.

Next step in our investigation will be the evaluation of the GLAID model using a case study, a 3D adventure game currently under development called Downtown: A subway Adventure. The game exploits playful aspects to improve the learning process of young people with Down syndrome and other impairments to increase their autonomy using the public transportation system. We are currently finishing the game and selecting the variables and data that researchers and educators will need to evaluate the effectiveness of the game design and the user learning experience. Once the development is finished, users will test the game, data will be collected and analyzed and future conclusions and lessons learned about the model will be released.

Acknowledgements. The e-UCM research group has been partially funded by Regional Government of Madrid (eMadrid S2013/ICE-2715), by the Ministry of Education (TIN2013-46149-C2-1-R) and by the European Commission (RAGE H2020-ICT-2014-1644187, BEACONING H2020-ICT-2015-687676). The CEIEC Institute has been partially funded by the Francisco de Vitoria University (UFV-2015-05).

\section{References}

1. Annetta, L., Minogue, J., Holmes, S., Cheng, M.: Investigating the impact of video games on high school students' engagement and learning about genetics. Comput. Educ. 53, 74-85 (2009) 
2. Griffiths, M.: The educational benefits of videogames. Educ. Health 20(3), 47-51 (2002)

3. Campbell, D.: Xbox Data is XXL. In: Strata Conference 2013, Santa Clara, California, 28 February 2013. https://www.youtube.com/watch?v=ZLKf1AhLQ30\&feature=youtu.be. Accessed 10 Mar 2016

4. Long, P., Siemenes, G.: Penetrating the fog: analytics in learning and education. In: 1st International Conference on Learning Analytics and Knowledge, Banff, Alberta, Canada (2011)

5. Freire, M., Serrano-Laguna, A., Manero, B., Martínez-Ortiz, I., Moreno-Ger, P., Fernández-Manjón, B.: Game learning analytics. In: Learning Analytics for Serious Games (2015)

6. Roberts, J., Price, J., Malkin, C.: Language and communication development in down syndrome. Ment. Retard. Dev. Disabil. Res. Rev. Spec. Issue: Lang. Commun. 13(1), 26-35 (2007)

7. Gartner, Introduction to the Gartner Maturity Model for Web Analytics, 03 July 2008. https://www.gartner.com/doc/713210/introduction-gartner-maturity-model-web. Accessed 03 Mar 2016

8. Australian Government. Department of Families, Housing, Community Services and Indigenous Affairs, "Intellectual disabilities, communication and learning", 20 January 2009. http://resources.fahcsia.gov.au/consumertrainingsupportproducts/employers/intellectual_ disability/sec3.htm. Accessed 11 Mar 2016

9. Arranz, P.: Niños y jóvenes con Síndrome de Down, Egido Editorial (2002)

10. Troncoso, M., Jesús, F.: Síndrome de Down: Avances en acción familiar, Santander: Fundación Síndrome de Down de Cantabria (1988)

11. Chapman, R., Hesketh, L.: Behavioral phenotype of individuals with Down Syndrome. Ment. Retard. Dev. Disabil. Res. Rev. 6(2), 84-95 (1999)

12. W3C World Wide Web Consortium, "WCAG 2.0." http://www.w3.org/TR/WCAG20/. Accessed 09 Mar 2016

13. Ablegamers Foundation, "Game Accessibililty Guidelines," 2012-2015. http://gameaccessi bilityguidelines.com/. Accessed 09 Mar 2016

14. Serrano-Laguna, A., Torrente, J., Moreno-Ger, P., Fernández-Manjón, B.: Tracing a little for big improvements: application of learning anañytics and videogames for student assessment. Procedia Comput. Sci. 15, 203-209 (2012)

15. Nyce, C.: Predictive Analytics White Paper, American Institute for Chartered Property Casualty Underwriters/Insurance Institute of America (2007) 